\title{
Association of Statins With Functional Outcome and 30-Day Mortality in Patients With Intracerebral Hemorrhage
}

Joana Silva Marques ${ }^{1}$, Giovana Ennis ${ }^{1}$, Gabriela Venade ${ }^{1}$, Rita João Soares ${ }^{1}$, Nuno Monteiro ${ }^{1}$, Ana Gomes ${ }^{2}$

1. Internal Medicine, Centro Hospitalar Tondela-Viseu, Viseu, PRT $\quad$ 2. Stroke Unit, Centro Hospitalar Tondela-Viseu, Viseu, PRT

Corresponding author: Joana Silva Marques, joanasilvamarques89@gmail.com

\section{Abstract}

\section{Aim}

The effect of statins is well established in cardiac and cerebrovascular diseases. However, its impact on intracerebral hemorrhage (ICH) is unclear. We aim to identify an association of pre-ICH statin treatment and statin use during admission for ICH with functional outcome at discharge and 30-day mortality.

\section{Material and methods}

A retrospective cohort study was held in patients with ICH admitted to our stroke unit over a year period. Demographic characteristics, risk factors and cardiovascular diseases, Glasgow Coma Scale (GCS), National Institutes of Health Stroke Score (NIHSS), systolic blood pressure (SBP) at admission, cholesterol levels and radiologic findings were analyzed to explore the association between pre-ICH and inpatient statin use with outcomes. The primary endpoint was functional outcome defined as modified Rankin Score (mRS) at discharge and 30-day mortality. We performed a univariate analysis and the variables with statistical significance were included in a multivariate analysis to control for confounding covariates.

\section{Results}

The study included 78 patients, 33 (42.31\%) had previous statin intake history, of which 13 (39.39\%) maintained statin intake during hospitalization. Regarding functional outcome we did not report a statistically significant difference between groups. In the "pre-ICH statin use" group a decreased 30-day mortality $(6.06 \%, p=0.009)$ was observed. In this group it was also noted higher antiplatelet medication use (33.33\%, $\mathrm{p}=0.006)$, higher GCS at admission $(13-15: 84.38 \%, \mathrm{p}=0.018)$ and deep ICH $(81.82 \%, \mathrm{p}=0.030)$. However, 30-day mortality had no impact in multivariate regression (Odds ratio (OR) $4.535,95 \%$ Confidence Interval $(\mathrm{CI})=0.786-26.173, \mathrm{p}=0.091)$. In the group that maintained statin treatment during hospitalization no deaths were registered $(p=0.020)$ and there was no association with functional status. Multivariate regression analysis was not performed due to sample size.

Review began 03/30/2021 Review ended 04/07/2021 Published 04/11/2021

\section{() Copyright 2021}

Silva Marques et al. This is an open access article distributed under the terms of the Creative Commons Attribution License CC-BY 4.0., which permits unrestricted use, distribution, and reproduction in any medium, provided the original author and source are credited.

\section{Conclusion}

The only association demonstrated in this study was lower 30-day mortality with pre-ICH statin use and continued statin treatment during admission. However, this was not confirmed by multivariate regression analysis. There were no differences between groups concerning cholesterol values, results that can be explained by the pleiotropic and immunomodulatory effect of statins. However, prospective studies are needed to prove the benefit of the statins in ICH.

Categories: Internal Medicine, Neurology

Keywords: intracerebral hemorrhage, hemorrhagic stroke, statins, mortality, modified rankin score

\section{Introduction}

Intracerebral hemorrhage (ICH) is the second most common cause of stroke (10-15\% of all strokes) [1]. The 30-day mortality ranges from $35 \%$ to $52 \%$ and half of these deaths occur within the first two days [2]. Given the morbidity and mortality associated with ICH, assessment of therapies with neuroprotective effects is of increasing interest.

Statins inhibit, in a reversible way, the HMG-CoA reductase, which is the limiting step in cholesterol synthesis [3]. This mechanism is responsible for dyslipidemia treatment and the reduction of primary and secondary cardiovascular events [3]. Despite these benefits, a post hoc analysis from Stroke Prevention by Aggressive Reduction in Cholesterol Levels (SPARCL) trial showed a three-fold increase of recurrent ICH in patients treated with statin [4]. There are data regarding the contribution of low levels of serum cholesterol 
to the cerebrovascular endothelium fragility and statin treatment to the decreased platelet aggregation and thrombogenesis. Both mechanisms could promote hematoma expansion, increase the risk for recurrent hemorrhage, and aggravate ICH mortality or functional outcomes [5-6].

Statins also have pleotropic effects, like anti-inflammatory, antithrombotic, antioxidative, and neuroprotective. These are being demonstrated in animal and human models [3]. In this regard, a few studies reported that statin use was associated with reduced mortality and improved functional outcome after ICH. There is a paucity of data regarding beneficial impact of stating in acute ICH.

Therefore, we set out to investigate the association between pre-ICH statin treatment and statin use during admission with functional outcomes and 30-day mortality.

This article was previously presented as a meeting poster at the 26th National Congress of Internal Medicine of the Portuguese Society of Internal Medicine on August 27, 2020.

\section{Materials And Methods}

We performed a retrospective study on primary ICH patients admitted in a single-center stroke unit, from 1st of June 2018 to the 31st of May 2019. All patients with a baseline admission computerized tomography (CT) scan and medical records of pre-ICH statin use were eligible. Individuals with no inclusion criteria, diagnosed with subdural or subarachnoid hemorrhage, ischemic stroke with hemorrhagic transformation, traumatic hemorrhage, bleeding cerebral tumor and cerebral vascular malformation were excluded.

Clinical information, which included chronic medication, was obtained from patients during the medical interview and complemented by medical records. Medical record review data included: pre-ICH statin use, statin treatment during hospitalization, age, gender, prior history of hypertension, diabetes mellitus (DM), cerebral vascular disease (CVD), body mass index (BMI), previous antiplatelet or anticoagulant treatment. Glasgow Coma Scale (GCS) and National Institutes of Health Stroke Score (NIHSS) at admission were considered and categorized according to cut-off points (GCS: 4-8, 9-12, 13-15; NIHSS: 0-4, 5-8, 9-16, 16-24) and CT characteristics were pointed out like hematoma locations, presence of intra-ventricular hematoma extension and volume. ICH location was categorized into lobar, deep, cerebelous and brainstem and volume calculated by A · B · C/ 2 method. Total cholesterol, low density lipoprotein (LDL), high density lipoprotein (HDL) and triglycerides level were also recorded.

Statin use was further dichotomized into prior-ICH use and no use and continued or discontinued during hospitalization.

The primary endpoints were the effect of statin use on functional status at discharge and 30-day mortality in ICH patients. Functional status was defined by modified Rankin Score (mRS) considering good outcome 0-2 and bad outcome 3-6.

Statistical analysis was performed in two steps. First, a univariate analysis was done. Statistically significant results were used in a multivariate regression analysis to explore the effect of pre-ICH statin use and statin continuation during hospitalization in functional outcome at discharge and 30-day mortality.

Categorical variables are presented as frequencies and percentages, and continuous variables as median and interquartile range due to skewed distributions or mean and standard deviation. Normal distribution was checked using Shapiro-Wilk test. Categorical variables were compared with chi-square test and continuous variables were compared with Mann-Whitney or t-student in non- or parametric test respectively. All reported "p" values are two-tailed, with a "p" value of 0.05 indicating statistical significance (CI 95\%). Analyses were performed with the use of "Statistical Package for the Social Sciences” (SPSS) version 26 (IBM Corp., Armonk, NY).

\section{Results}

Eighty-three ICH patients were admitted in the stroke unit over the study period. Five cases were excluded due to missing data about pre-ICH statin use.

Our final study population included 78 patients, of which $57.70 \%$ (45) were male. Median age was $76.00+/-$ 14.00 years, a median length of stay in the stroke unit was $4.24+/-2.35$ days and $17.35+/-20.74$ days in other medical wards. Overall, 11 patients $(14.10 \%)$ had good functional outcome and 16 (20.51\%) patients died during the first 30 days after ICH, of which 10 (62.50\%) occurred in stroke unit and six (37.50\%) in other wards.

Thirty-three (43.31\%) had medical history of statin use and 45 (57.69\%) had no pre-ICH statin use. In the statin user group, 13 (39.40\%) continued statins and 20 (60.61\%) discontinued statin during admission.

Table 1 compares the baseline characteristics and outcomes of interest among statin and non-statin users, 


\section{Cureus}

respectively. The univariate analysis showed with statistical significance that "pre-ICH statin" group was associated to a higher number of patients with antiplatelets use $(33.33 \%, \mathrm{p}=0.006)$, higher GCS at admission (13-15: $84.38 \%, p=0.018)$ and deep hemorrhage, with no cases of cerebellum or brainstem hemorrhage $(81.82 \%, \mathrm{p}=0.030)$ (Table 1). Although no statistical significance was found, in this group the patients were older $(78.00+/-11.50, \mathrm{p}=0.451)$, had more hypertension $(78.10 \%, \mathrm{p}=0.087)$ and CVD $(36.36 \%, \mathrm{p}=0.060)$ and higher BMI $(29.40+/-6.06, \mathrm{p}=0.50)$. This same group was associated with, NIHSS at admission 0-4 and 9-16 $(25.00 \%, 45.00 \%$, respectively, $\mathrm{p}=0.189)$, lower systolic blood pressure $(\mathrm{SBP})(166.42+/-61.57, \mathrm{p}=0.778)$ smaller hemorrhage volume on head CT $(12.80+/-57.20, \mathrm{p}=0.295)$ and fewer cases of intra-ventricular hematoma $(42.42 \%, \mathrm{p}=0.819)$. The "pre-ICH statin" group had better cholesterol levels with lower total cholesterol (TC) $(153.00+/-43.00, \mathrm{p}=0.286)$, lower low density lipoprotein (LDL) $(85.40+/-35.10, \mathrm{p}=0.58)$, lower triglycerides (TG) $(105.35+/-47.93, \mathrm{p}=0.582)$ and higher high density lipoprotein (HDL) $(46.10+/-$ $10.50, \mathrm{p}=0.286)$. 


\section{Cureus}

\begin{tabular}{|c|c|c|c|c|}
\hline & Total number cases, $n=78$ & Pre-ICH statin, $n=33$ & No pre-ICH statin, $n=45$ & p \\
\hline Age, years & $76.00+/-14.00$ & $78.00+/-11.50$ & $76.00+/-18.00$ & 0.451 \\
\hline Gender, male & $45(57.69)$ & $18(54.55)$ & $27(60.00)$ & 0.650 \\
\hline HT & $51(66.20)$ & $25(78.0)$ & $26(57.80)$ & 0.087 \\
\hline DM & $33(42.31)$ & $14(42.42)$ & $19(42.22)$ & 1.000 \\
\hline CVD & 19 (24.36) & $12(36.36)$ & $7(15.56)$ & 0.060 \\
\hline BMI, kg/m² & $27.24+/-4.53$ & $29.40+/-6.06$ & $26.42+/-5.66$ & 0.50 \\
\hline OAC & 14 (17.95) & $8(24.24)$ & $6(13.33)$ & 0.244 \\
\hline Antiplatelets & 14 (17.95) & $11(33.33)$ & $3(6.67)$ & 0.006 \\
\hline TC (mg/dL) & $161.00+/-47.00$ & $153.00+/-43.00$ & $169.50+/-73.00$ & 0.286 \\
\hline LDL (mg/dL) & $93.15+/-37.08$ & $85.40+/-35.10$ & $102.50+/-45.35$ & 0.58 \\
\hline HDL (mg/dL) & $44.80+/-11.90$ & $46.10+/-10.50$ & $40.00+/-15.90$ & 0.353 \\
\hline TG (mg/dL) & $101.80+/-61.50$ & $105.35+/-47.93$ & $107.25+/-80.85$ & 0.582 \\
\hline \multicolumn{5}{|l|}{ NIHSS admission } \\
\hline $0-4$ & $7(17.50)$ & $5(25.00)$ & $2(10.00)$ & \multirow{4}{*}{0.189} \\
\hline 5-8 & $9(22.50)$ & $4(20.00)$ & $5(25.00)$ & \\
\hline 9-16 & $15(37.50)$ & $9(45.00)$ & $6(30.00)$ & \\
\hline $17-24$ & $9(22.50)$ & $2(10.00)$ & $7(35.00)$ & \\
\hline \multicolumn{5}{|l|}{ GCS admission } \\
\hline $4-8$ & $5(6.49)$ & $1(3.13)$ & $4(8.89)$ & \multirow{3}{*}{0.018} \\
\hline $9-12$ & $21(27.27)$ & $4(12.50)$ & $17(37.78)$ & \\
\hline 13-15 & $51(66.23)$ & $27(84.38)$ & $24(53.33)$ & \\
\hline SBP (mmHg) & $167.68+/-32.22$ & $166.42+/-61.57$ & $168.58+/-33.02$ & 0.778 \\
\hline \multicolumn{5}{|l|}{ ICH location } \\
\hline Lobar & $23(29.49)$ & $6(18.18)$ & $17(37.78)$ & \multirow{4}{*}{0.030} \\
\hline Deep & $50(64.10)$ & 27 (81.82) & $23(51.11)$ & \\
\hline Cerebellum & $3(3.75)$ & $0(0)$ & $3(6.67)$ & \\
\hline Brainstem & $2(2.56)$ & $0(0)$ & $2(4.44)$ & \\
\hline Volume $\left(\mathrm{mm}^{3}\right)$ & $21.00+/-52.30$ & $12.80+/-57.20$ & $24.00+/-48.30$ & 0.295 \\
\hline Intra-ventricular hematoma & $35(44.87)$ & 14 (42.42) & $21(46.67)$ & 0.819 \\
\hline \multicolumn{5}{|l|}{ mRS discharge } \\
\hline $0-2$ & $11(14.10)$ & $7(21.21)$ & $4(8.89)$ & \multirow[t]{2}{*}{0.176} \\
\hline 3-6 & 34 (43.59) & $13(39.40)$ & $21(46.67)$ & \\
\hline 30-day mortality & $16(20.51)$ & $2(6.06)$ & $14(31.11)$ & 0.009 \\
\hline
\end{tabular}

\section{TABLE 1: Univariate analysis of different variables and association with pre-ICH statin use}

BMI: Body Mass Index; CVD: Cerebral Vascular Disease; DM: Diabetes mellitus; GCS: Glasgow Coma Scale; HDL: High Density Lipoprotein; HT: Hypertension; ICH: Intracerebral hemorrhage; LDL: Low Density Lipoprotein; mRS: modified Rankin Score; NIHSS: NIH Stroke Scale; OAC: Oral Anticoagulants; SBP: Systolic Blood Pressure; TC: Total Cholesterol; TG: Triglycerides. 


\section{Cureus}

Comparing with primary outcomes there was no significant differences between groups in functional outcome at discharge $(\mathrm{p}=0.176)$ but a lower mortality was observed in the "pre-ICH statin" group with statistical significance $(6.06 \%$ vs $31.11 \%, p=0.009)$ (Table 1$)$. In multivariate analysis (Table 2$)$, the group of pre-ICH statin use did not show any significant impact in 30-day mortality after adjusting to other variables (OR 4.535, 95\% CI 0.786-26.173, p = 0.091).

\begin{tabular}{|c|c|c|c|}
\hline & OR & $\mathbf{p}$ & Cl \\
\hline Antiplatelets & 0.850 & 0.873 & 0.117-6.168 \\
\hline GCS & 5.830 & 0.001 & 2.005-16.949 \\
\hline $\mathrm{ICH}$ location & 1.023 & 0.963 & $0.391-2.679$ \\
\hline Pre-ICH statin & 4.535 & 0.091 & $0.786-26.173$ \\
\hline
\end{tabular}

TABLE 2: Multivariate analysis of pre-ICH use and 30-day mortality

Cl: Confidence interval; GCS: Glasgow Coma Score; ICH: Intracerebral Hemorrhage; OR: Odds Ratio

A significant association between fewer CVD history $(23.08 \%, \mathrm{p}=0.038)$, more antiplatelets use $(38.46 \%, \mathrm{p}=$ $0.008)$, lower NIHSS at admission (0-4: 30.77\% and 9-16: $30.77 \%, \mathrm{p}=0.05)$ and higher GCS $(13-15: 100 \%, \mathrm{p}=$ 0.033 ) was observed in the continued statin therapy during admission group (Table 3). Regarding functional outcome we did not register statistical differences between groups $(\mathrm{p}=0.282)$. No deaths were observed in the group that continued statin therapy during admission and only two in the group that discontinued therapy. This data did not fit our previous model.

\begin{tabular}{|c|c|c|c|c|}
\hline & $\begin{array}{l}\text { Pre-ICH statin, } n \\
=33\end{array}$ & $\begin{array}{l}\text { Continued statin therapy during } \\
\text { admission, } n=13\end{array}$ & $\begin{array}{l}\text { Discontinued statin therapy during } \\
\text { admission, } n=20\end{array}$ & p \\
\hline Age, years & $78.00+/-11.50$ & $79.00+/-14.50$ & $77.50+/-9.50$ & 0.748 \\
\hline Gender, male & 18 (54.55) & $7(53.84)$ & $11(55.00)$ & 0.889 \\
\hline HT & $51(66.20)$ & 10 (76.92) & $15(75.00)$ & 0.158 \\
\hline DM & 33 (42.31) & $5(38.46)$ & $9(45.00)$ & 0.933 \\
\hline CVD & $19(24.36)$ & $3(23.08)$ & $9(45.00)$ & 0.038 \\
\hline BMI, kg/m² & $27.24+/-4.53$ & $29.40+/-8.09$ & $28.96+/-6.40$ & 0.141 \\
\hline OCA & 14 (17.95) & $3(23.08)$ & $5(25.00)$ & 0.459 \\
\hline Antiplatelets & 14 (17.95) & $5(38.46)$ & $6(30.00)$ & 0.008 \\
\hline TC (mg/dL) & $161.0+/-47.00$ & $158.00+/-91.75$ & $151.00+/-42.00$ & 0.356 \\
\hline LDL (mg/dL) & $93.15+/-37.08$ & $93.00+/-82.37$ & $85.75+/-37.85$ & 0.105 \\
\hline HDL (mg/dL) & $44.80+/-11.90$ & $44.85+/-10.88$ & $46.75+/-10.40$ & 0.640 \\
\hline TG (mg/dL) & $101.80+/-61.50$ & $112.10+/-41.45$ & $105.35+/-59.70$ & 0.801 \\
\hline \multicolumn{5}{|c|}{ NIHSS admission } \\
\hline $0-4$ & $7(17.50)$ & $4(30.77)$ & $1(5.00)$ & \multirow{4}{*}{0.05} \\
\hline $5-8$ & $9(22.50)$ & $0(0)$ & $4(20.00)$ & \\
\hline $9-16$ & $15(37.50)$ & $4(30.77)$ & $5(25.00)$ & \\
\hline $17-24$ & $9(22.50)$ & $0(0)$ & $2(10.00)$ & \\
\hline \multicolumn{5}{|l|}{ GCS admission } \\
\hline 4-8 & $5(6.49)$ & $0(0)$ & $1(5.00)$ & \multirow{2}{*}{0.033} \\
\hline $9-12$ & $21(27.27)$ & $0(0)$ & $4(20.00)$ & \\
\hline
\end{tabular}




\section{Cureus}

\begin{tabular}{|c|c|c|c|c|}
\hline $13-15$ & $51(66.23)$ & $13(100.00)$ & $14(70.00)$ & \\
\hline SBP (mmHg) & $167.68+/-32.22$ & $169.00+/-25.81$ & $164.56+/-35.77$ & 0.706 \\
\hline \multicolumn{5}{|l|}{ ICH location } \\
\hline Lobar & $23(29.49)$ & $3(23.08)$ & $3(15.00)$ & \multirow{4}{*}{0.163} \\
\hline Deep & $50(64.10)$ & $10(76.92)$ & $17(85.00)$ & \\
\hline Cerebellum & $3(3.75)$ & $0(0)$ & $0(0)$ & \\
\hline Brainstem & $2(2.56)$ & $0(0)$ & $0(0)$ & \\
\hline Volume $\left(\mathrm{mm}^{3}\right)$ & $21.00+/-52.30$ & $16.05+/-50.45$ & $14.55+/-53.83$ & 0.343 \\
\hline $\begin{array}{l}\text { Intra-ventricular } \\
\text { hematoma }\end{array}$ & $35(44.87)$ & 2 (15.38) & $12(60.00)$ & 0.039 \\
\hline 30-day mortality & $16(20.51)$ & $0(0)$ & $2(10.00)$ & 0.020 \\
\hline \multicolumn{5}{|l|}{ mRS discharge } \\
\hline $0-2$ & $11(14.10)$ & $3(23.08)$ & $4(20.00)$ & \multirow[t]{2}{*}{0.282} \\
\hline $3-6$ & 34 (43.59) & $4(30.77)$ & $9(45.00)$ & \\
\hline
\end{tabular}

TABLE 3: Univariate analysis of different variables and association with continued statin treatment during admission

BMI: Body Mass Index; CVD: Cerebral Vascular Disease; DM: Diabetes mellitus; GCS: Glasgow Coma Scale; HDL: High Density Lipoprotein; HT: Hypertension; ICH: Intracerebral Hemorrhage; LDL: Low Density Lipoprotein; mRS: modified Rankin Score; NIHSS: NIH Stroke Scale; OAC: Oral Anticoagulants; SBP: Systolic Blood Pressure; TC: Total Cholesterol; TG: Triglycerides.

\section{Discussion}

In our population, pre-ICH statin treatment and statin use during hospitalization was not associated with functional outcomes at discharge. A trend associating statin use pre-ICH or during admission with decreased 30-day mortality was noted with statistical significance but with no impact after adjusting for other potential confounders.

The findings of our study shed light on the contradictory data about the influence of statins on outcomes after ICH. This is a complex disease and involves several factors that independently or co-dependently affect the outcome of these patients.

In 2004, SPARCL trial found out that treatment with atorvastatin was independently associated with an increased risk of hemorrhagic stroke (hazard ratio, 1.68; 95\% CI, 1.09-2.59) [4]. However, this trial enrolled patients with prior ischemic stroke and with probable secondary microvascular injury [3].

There are studies that reported increased rates of ICH and ICH-related mortality in patients with low cholesterol levels [7] and that low LDL was an important predictor of hematoma growth [6]. It has been hypothesized that cholesterol may be important for cerebrovascular wall integrity and that low levels may increase the risk of vessel rupture and higher fibrinolytic activity [8]. Statins are lipid-lowering therapy so we should expect a bad outcome in patients with pre-ICH statin use [7].

In our study, the results were independent of cholesterol levels. It was also noted an association between pre-ICH statin use and better GCS at admission. Hence, the clinical benefit may not be limited to the lipidlowering properties of statins but also derived from other "pleiotropic" effects [7]. In animal experiments it has been possible to demonstrate the capacity of statins to improve ICH mortality by the promotion of neuronal plasticity and the limitation of damage in the boundary tissue [9], the increase of cerebral perfusion (mediated via increased endothelial nitric oxide secretion resulting in vasodilatation), the antiinflammatory proprieties, the improvement of angiogenesis and neurogenesis after ischemic injury. There is also evidence that statin discontinuation could lead to a rebound effect resulting in oxidative stress and vascular disfunction [10].

Supporting these findings, many observational studies in humans were performed, but with contradictory findings (Table 4). In 2007, Naval et al.'s study was the first retrospective study that reported the benefit of pre-ICH statins treatment in humans by reducing early absolute edema volume and decreasing 30-day mortality (OR 0.080, $\mathrm{p}=0.050$ ), with no effect in functional outcomes at discharge [11]. FitzMaurice et al. 
found no effect of pre-ICH statin use on the rates of functional independence (28\% versus $29 \%$, P 0.84 ) or mortality ( $46 \%$ versus $45 \%$, P 0.93 ) and ICH survivors treated with statins after discharge did not have a higher risk of recurrence (adjusted HR 0.82, 95\% CI 0.34-1.99, P 0.66) [11]. However cholesterol and triglycerides levels were not reported in any group, and medical comorbidity was more common in statin pretreated patients than in the non-pretreated group. Eichel et al. found that despite having significantly smaller haematomas, patients that were using statins did not have better neurological or functional outcomes at 90-day post-ICH [12]. Statin users more often had existing co-morbidities that adversely affect outcomes. This data points to the fact that statin users represent a generally more impaired population, and that these comorbidities can counteract the possible beneficial effects of statin use. Flint et al. designed a multicentric retrospective study and reported an increased mortality in the group that discontinued statin during hospitalization. Multivariable regression showed a relationship between statin use and higher probability of 30-day survival ( 0.15 [95\% CI, 0.04-0.25]; $\mathrm{P}=.01$ ). Winkler et al. had a longer follow-up (12 months) and concluded that pre-ICH statin group was associated with decreased mortality in-hospital and 12 months $(\mathrm{p}=0.001)$ confirmed after multivariable adjustments [13].

\begin{tabular}{|c|c|c|c|c|c|}
\hline & Study type & Cohort & Exclusions & End-points & Results \\
\hline $\begin{array}{l}\text { Naval et al., } \\
2007 \text { [11] }\end{array}$ & Retrospective & $\begin{array}{l}125 \\
26 \% \\
\text { statin }\end{array}$ & $\begin{array}{l}\text { Trauma, } \\
\text { cerebral } \\
\text { tumour, } \\
\text { aneurisms, } \\
\text { AVM, } \\
\text { infratentorial } \\
\text { ICH }\end{array}$ & $\begin{array}{l}\text { 30-day } \\
\text { mortality }\end{array}$ & $\begin{array}{l}\text { Multiple logistic regression analysis, prior statin use }(P=0.05) \text { was } \\
\text { found to be associated with decreased mortality with a greater than } \\
12 \text {-fold odds of survival }\end{array}$ \\
\hline $\begin{array}{l}\text { FitzMaurice } \\
\text { et al., } 2008 \\
\text { [12] }\end{array}$ & Retrospective & $\begin{array}{l}629 \\
24 \% \\
\text { statin }\end{array}$ & $\begin{array}{l}\text { Secondary } \\
\mathrm{ICH}, \text { no } 90 \text {-day } \\
\text { (GOS) } 90 \text { days }\end{array}$ & $\begin{array}{l}\text { Good 90-day } \\
\text { outcome (GOS } \\
4-5)\end{array}$ & $\begin{array}{l}\text { No effect of pre-ICH statin use on the rates of functional } \\
\text { independence }(28 \% \text { versus } 29 \%, \mathrm{P} 0.84 \text { ) or mortality ( } 46 \% \text { versus } \\
45 \%, \mathrm{P} 0.93) \text {. Multivariable analysis for independent status in pre- } \\
\mathrm{ICH} \text { statin users was } 1.16 \text { ( } 95 \% \mathrm{CI} 0.65 \text { to } 2.10, \mathrm{P} 0.62) . \mathrm{ICH} \text { survivors } \\
\text { treated with statins after discharge did not have a higher risk of } \\
\text { recurrence (adjusted } \mathrm{HR} 0.82,95 \% \mathrm{Cl} 0.34 \text { to } 1.99, \mathrm{P} 0.66) \text {. }\end{array}$ \\
\hline $\begin{array}{l}\text { Leker et al., } \\
2009 \text { [14] }\end{array}$ & Retrospective & $\begin{array}{l}312, \\
28,5 \% \\
\text { statin }\end{array}$ & Secondary ICH & $\begin{array}{l}\text { Good } \\
\text { functional } \\
\text { outcome at } \\
\text { discharge } \\
\text { (mRS 0-3). } \\
\text { Discharge at } \\
\text { nursery facility, } \\
\text { home or death }\end{array}$ & $\begin{array}{l}\text { Pre-ICH statin had higher proportions of mRS } 0-3 \text {, lower death rates, } \\
\text { and higher rates of discharge home or to a rehabilitation facility. } \\
\text { Logistic regression analyses of pre-ICH statin OR } 2.97 \text { for mRS } 0-3 \\
(95 \% \mathrm{Cl} ; 1.25 \text { to } 7.35 \text { ) at discharge and OR } 0.25 \text { for death or nursing } \\
\text { facility disposition ( } 95 \% \mathrm{Cl} ; 0.09 \text { to } 0.63 \text { ). }\end{array}$ \\
\hline $\begin{array}{l}\text { Eichel et al., } \\
2010 \text { [13] }\end{array}$ & Retrospective & $\begin{array}{l}399, \\
25,3 \% \\
\text { statin }\end{array}$ & $\begin{array}{l}\text { (hemorrhagic } \\
\text { transformation } \\
\text { from stroke, } \\
\text { tumor, AVM), } \\
\text { SAH, SDH }\end{array}$ & $\begin{array}{l}90 \text {-day } \\
\text { mortality. Good } \\
90 \text {-day } \\
\text { functional } \\
\text { outcome (mRS } \\
<2 \text { ) }\end{array}$ & $\begin{array}{l}\text { Pre-ICH statin had no association with primary endpoints } \mathrm{mRS}<2 \text { or } \\
\text { mortality. No impact on multiple logistic regression analysis. }\end{array}$ \\
\hline $\begin{array}{l}\text { Gomis et } \\
\text { al., } 2010 \\
{[15]}\end{array}$ & Retrospective & $\begin{array}{l}269, \\
12.6 \% \\
\text { statin }\end{array}$ & $\begin{array}{l}\text { Secondary } \\
\mathrm{ICH}, \mathrm{ICH} \text { due } \\
\text { to } \\
\text { anticoagulation } \\
\text { therapy, } \\
\text { primary IVH, } \\
\text { mRS basal >1 }\end{array}$ & $\begin{array}{l}\text { Good 90-day } \\
\text { functional } \\
\text { outcome (mRS } \\
0-2 \text { ) }\end{array}$ & $\begin{array}{l}\text { Multivariate regression analysis showed a significant association } \\
\text { between age (OR: } 0.95 \text {; } \mathrm{Cl} 0.92-0.97), \mathrm{ICH} \text { volume (OR: } 0.96 \text {; } \mathrm{Cl} 0.94- \\
0.98) \text {, GCS (OR: } 1.55 \text {; } \mathrm{Cl} 1.21-1.98) \text {, pre-treatment with statins (OR: } \\
4.21 \text {; Cl 1.47-12.17; } \mathrm{P}=0.008) \text {, and mRS } 0-2 \text { at } 3 \text { months. }\end{array}$ \\
\hline $\begin{array}{l}\text { Biffi et al., } \\
2011 \text { [1] }\end{array}$ & Retrospective & $\begin{array}{l}699, \\
34.0 \% \\
\text { statin }\end{array}$ & Secondary ICH & $\begin{array}{l}\text { Good } 90 \text {-day } \\
\text { functional } \\
\text { outcome (mRS } \\
\text { 0-2) } 90 \text {-day } \\
\text { mortality }\end{array}$ & $\begin{array}{l}\text { Association between statin use before } \mathrm{ICH} \text { and increased probability } \\
\text { of favorable outcome (OR } 2.08,95 \% \mathrm{Cl} 1.37-3.17 \text { ). Reduced } \\
\text { mortality (OR } 0.47,95 \% \mathrm{Cl} 0.32-0.70 \text { ) at } 90 \text { days. Meta-analysis of all } \\
\text { published evidence confirmed the effect of statin use on good } \\
\text { outcome (OR } 1.91,95 \% \mathrm{Cl} 1.38-2.65 \text { ) and mortality (OR } 0.55,95 \% \mathrm{Cl} \\
0.42-0.72 \text { ) after ICH }\end{array}$ \\
\hline $\begin{array}{l}\text { Romero et } \\
\text { al., } 2011 \\
{[16]}\end{array}$ & Prospective & $\begin{array}{l}83, \\
24 \% \\
\text { statin }\end{array}$ & $\begin{array}{l}\text { Secondary } \\
\mathrm{ICH}, \text { No CT at } \\
\text { admission, no } \\
\text { important data }\end{array}$ & $\begin{array}{l}\text { 90-day } \\
\text { outcome (GOS) } \\
90 \text {-day } \\
\text { mortality }\end{array}$ & $\begin{array}{l}\text { No effect from pre- } \mathrm{ICH} \text { statin use on the functional independence } \\
\text { rates }(32 \% \text { vs } 36 \%, \mathrm{P}=0.79) \text { or mortality }(41 \% \text { versus } 47 \%, \mathrm{P}=0.82) \text {. }\end{array}$ \\
\hline
\end{tabular}




\section{Cureus}

\begin{tabular}{|c|c|c|c|c|c|}
\hline $\begin{array}{l}\text { Dowlatshahi } \\
\text { et al., } 2012 \\
\text { [17] }\end{array}$ & Retrospective & $\begin{array}{l}2466, \\
21.8 \% \\
\text { statin }\end{array}$ & Secondary ICH & $\begin{array}{l}\text { at discharge } \\
\text { (mRS 4-6) } 30- \\
\text { day and } 180- \\
\text { day mortality }\end{array}$ & $\begin{array}{l}\text { admission, had poor outcome }(90 \% \text { vs } 62 \%, \mathrm{P} 0.01) \text {, and higher } 30 \text { - } \\
\text { day mortality }(71 \% \text { vs } 21 \%, \mathrm{P} 0.01) \text {. After adjusting for stroke } \\
\text { severity, statin discontinuation was still associated with poor } \\
\text { outcome (adjusted OR, } 2.4 ; 95 \% \mathrm{Cl}, 1.13-4.56 \text { ) and higher mortality } \\
\text { (adjusted OR, } 2.0 ; 95 \% \mathrm{Cl}, 1.30-3.04) \text {. }\end{array}$ \\
\hline $\begin{array}{l}\text { King et al., } \\
2012[18]\end{array}$ & Prospective & $\begin{array}{l}1381, \\
21.1 \% \\
\text { statin }\end{array}$ & $\begin{array}{l}\text { Trauma, } \\
\text { Secondary } \\
\text { ICH, stroke, } \\
\text { tumor, AVM, } \\
\text { ICH due to } \\
\text { anticoagulant } \\
\text { therapy, no } \\
\text { data regarding } \\
\text { previous } \\
\text { medication }\end{array}$ & $\begin{array}{l}\text { 30-day } \\
\text { mortality }\end{array}$ & $\begin{array}{l}\text { Multivariate logistic regression did not demonstrate any effect of } \\
\text { prior statin use }(p=0.781) \text { on mortality. }\end{array}$ \\
\hline $\begin{array}{l}\text { Mustanoja } \\
\text { et al., } 2013 \\
\text { [19] }\end{array}$ & Retrospective & $\begin{array}{l}964, \\
19 \% \\
\text { statin }\end{array}$ & & $\begin{array}{l}\text { Good } \\
\text { functional } \\
\text { outcome (mRS } \\
\leq 2 \text { ). } \\
\text { Hospitalization, } \\
\text { 30-day and 90- } \\
\text { day mortality }\end{array}$ & $\begin{array}{l}\mathrm{mRS} \text { at discharge or mortality did not differ between groups (pre-ICH } \\
\text { statin use). Compared with survivors, significantly lower total } \\
\text { cholesterol and low-density lipoprotein cholesterol levels were } \\
\text { observed in patients who died in hospital (median, } 4.1 \mathrm{mmol} / \mathrm{L} \text { [IQR, } \\
3.6-4.4] \text { versus } 4.5 \text { [3.8-5.1). }\end{array}$ \\
\hline $\begin{array}{l}\text { Flint et al., } \\
2014 \text { [20] }\end{array}$ & Retrospective & $\begin{array}{l}3481, \\
34.3 \% \\
\text { statin }\end{array}$ & $\begin{array}{l}\text { Previous ICH, } \\
\text { not living } \\
\text { KPNC range, } \\
\text { no information } \\
\text { regarding } \\
\text { statin use }\end{array}$ & $\begin{array}{l}30 \text {-day } \\
\text { mortality. } \\
\text { Discharge to } \\
\text { home or } \\
\text { inpatient } \\
\text { rehabilitation } \\
\text { facility }\end{array}$ & $\begin{array}{l}\text { Inpatient statin users were more likely than nonusers to be alive } 30 \\
\text { days after ICH (OR } 4.25[95 \% \mathrm{Cl}, 3.46-5.23] ; \mathrm{P}<.001 \text { ) and were more } \\
\text { likely to be discharged to their home or an acute rehabilitation facility } \\
\text { (OR, } 2.57 \text { [ } 95 \% \mathrm{Cl}, 2.16-3.06] ; \mathrm{P}<.001 \text { ). Statin therapy was } \\
\text { discontinued, nonusers were less likely than statin users to survive to } \\
30 \text { days (OR, } 0.16 \text { [ } 95 \% \mathrm{Cl}, 0.12-0.21] ; \mathrm{P}<.001 \text { ) and were less likely } \\
\text { than statin users to be discharged to their home or an acute } \\
\text { rehabilitation facility (OR, } 0.26 \text { [ } 95 \% \mathrm{Cl}, 0.20-0.35] ; \mathrm{P}<.001 \text { ). } \\
\text { Multivariable regression showed that statin therapy was associated } \\
\text { with a higher probability of } 30 \text {-day survival (with an increase in } \\
\text { probability of } 0.15 \text { [95\% } \mathrm{Cl}, 0.04-0.25] ; \mathrm{P}=.01 \text { ) and a better chance } \\
\text { of being discharged to home or an acute rehabilitation facility (with } \\
\text { an increase in probability of } 0.13[95 \% \mathrm{Cl}, 0.02-0.24] ; \mathrm{P}=.02 \text { ) }\end{array}$ \\
\hline $\begin{array}{l}\text { Winkler et } \\
\text { al., } 2013 \\
\text { [21] }\end{array}$ & Retrospective & $\begin{array}{l}426, \\
44,6 \% \\
\text { statin }\end{array}$ & Secondary ICH & $\begin{array}{l}\text { Hospitalization } \\
\text { and 12-month } \\
\text { mortality. 12- } \\
\text { month blood } \\
\text { functional } \\
\text { outcome } \\
\text { (Barthel index) }\end{array}$ & $\begin{array}{l}\text { Pre-ICH statin group was associated with decreased mortality in- } \\
\text { hospital and } 12 \text { months ( } p=0.001) \text {. Multivariable analysis found a } \\
\text { decreased odds of death or disability at } 12 \text { months after ICH (OR } \\
0.44 ; 95 \% \mathrm{Cl} 0.21-0.95) \text {. }\end{array}$ \\
\hline $\begin{array}{l}\text { Siddiqui et } \\
\text { al., 2017 } \\
\text { [22] }\end{array}$ & Retrospective & $\begin{array}{l}2457, \\
10.9 \% \\
\text { statin }\end{array}$ & $\begin{array}{l}\text { Secondary } \\
\text { ICH, no data } \\
\text { regarding } \\
\text { functional } \\
\text { status or } \\
\text { hematoma } \\
\text { volume }\end{array}$ & $\begin{array}{l}\text { 90-day } \\
\text { mortality. Bad } \\
\text { 90-day } \\
\text { functional } \\
\text { outcome } \\
\text { (mRS) }\end{array}$ & $\begin{array}{l}\text { Statin use was associated with reduced mortality and disability } \\
\text { without any effect on hematoma growth. This association was } \\
\text { primarily driven by continued/new statin use. Multivariate analysis } \\
\text { showed continued/new statins users had good outcomes over prior } \\
\text { users. However, statins may have been continued/started more } \\
\text { frequently among less severe patients. Propensity score was } \\
\text { developed based on factors that could influence a physician's } \\
\text { decision in prescribing statins and used as a covariate, } \\
\text { continued/new statin use was no longer a significant predictor of } \\
\text { good outcome. }\end{array}$ \\
\hline
\end{tabular}

\section{TABLE 4: Clinical studies investigating the effects of statins on ICH}

AVM: Arteriovenous malformation; CT: Computerized Tomography; CI: Confidence Interval; CNS: Canadian Neurological Scale; GOS: Glasgow Outcome Scale; ICH: Intracerebral Hemorrhage; IQR: Interquartile Range; IVH: Intraventricular Hemorrhage; KPNC: Kaiser Permanente Northern California; mRS: modified Rankin Score; OR: Odds Ratio; SAH: Subarachnoid hemorrhage; SDH: Subdural hemorrhage. 
patients) and outcomes. Only two prospective studies were performed, and both did not observe any association between pre-ICH statin use and 30-day mortality.

A few limitations to our study should be pointed out. This is an observational retrospective study although data was collected prospectively. It is possible that unmeasured or unknown confounders may influence the results. However, multiple regression helped to reduce this potential bias. We had a small sample size which could decrease the power of our study and make it difficult to test whether any particular statin or dose had superior effects. There was a lack of information regarding some variables like BMI $(n=34)$, lipidic profile at admission $(n=43-46)$, NIHSS at admission $(n=40)$ and mRS at discharge $(n=45)$. The later was a limitation to investigate the association between pre-ICH statin use and continuation during hospitalization and functional outcome at discharge. Also, clinical outcomes (mRS and mortality) were evaluated at discharge and not in a prolonged subacute time frame to maximize clinical recovery after ICH. Nevertheless, we acknowledge that neurological recovery following stroke may take place over longer periods of time and the variability in the time period following ICH that these patients were discharged might affect the data assessing functional outcomes.

\section{Conclusions}

In our study, pre-ICH statin use or continued treatment during admission was not associated with improved functional status. However, an association between these groups and decreased 30-day mortality was reported. These findings did not persist after adjusting for other variables.

Preclinical and clinical studies support the potential neuroprotective and recovery enhancement effects afforded by statins in the setting of acute ICH. There are conflicting data influenced due to heterogeneity of retrospective studies and absence of large prospective trials evaluating the safety and efficacy of statin therapy in ICH. Considering that the interpretations of our results are restricted by several limitations, future studies considering the impact of statins on mortality and functional outcomes are needed.

\section{Additional Information \\ Disclosures}

Human subjects: Consent was obtained or waived by all participants in this study. Animal subjects: All authors have confirmed that this study did not involve animal subjects or tissue. Conflicts of interest: In compliance with the ICMJE uniform disclosure form, all authors declare the following: Payment/services info: All authors have declared that no financial support was received from any organization for the submitted work. Financial relationships: All authors have declared that they have no financial relationships at present or within the previous three years with any organizations that might have an interest in the submitted work. Other relationships: All authors have declared that there are no other relationships or activities that could appear to have influenced the submitted work.

\section{References}

1. Biffi A, Devan WJ, Anderson CD, et al.: Statin use and outcome after intracerebral hemorrhage: case-control study and meta-analysis. Neurology. 2011, 76:1581-1588. 10.1212/WNL.0b013e3182194be9

2. Moulin S, Cordonnier C: Prognosis and outcome of intracerebral haemorrhage. Front Neurol Neurosci. 2016, 37:182-192. 10.1159/000437122

3. Van Matre ET, Sherman DS, Kiser TH: Management of intracerebral hemorrhage--use of statins . Vasc Health Risk Manag. 2016, 12:153-161. 10.2147/VHRM.S75399

4. Amarenco P, Benavente O, Goldstein LB, et al.: Results of the Stroke Prevention by Aggressive Reduction in Cholesterol Levels (SPARCL) trial by stroke subtypes. Stroke. 2009, 40:1405-1409.

10.1161/STROKEAHA.108.534107

5. Konishi M, Iso H, Komachi Y, et al.: Associations of serum total cholesterol, different types of stroke, and stenosis distribution of cerebral arteries. The Akita Pathology Study. Stroke. 1993, 24:954-964. 10.1161/01.str.24.7.954

6. Lei C, Wu B, Liu M, Chen Y: Association between statin use and intracerebral hemorrhage: a systematic review and meta-analysis. Eur J Neurol. 2014, 21:192-198. 10.1111/ene.12273

7. McKinney JS, Kostis WJ: Statin therapy and the risk of intracerebral hemorrhage: a meta-analysis of 31 randomized controlled trials. Stroke. 2012, 43:2149-2156. 10.1161/STROKEAHA.112.655894

8. Jhuo SJ, Tsai WC, Lin TH, Voon WC, Lai WT, Sheu SH: Statin dose and the risk of intracerebral hemorrhage: a population-based longitudinal study in Taiwan. Acta Cardiol Sin. 2016, 32:23-30. 10.6515/acs20150204c

9. Tapia-Perez H, Sanchez-Aguilar M, Torres-Corzo JG, Rodriguez-Leyva I, Gonzalez-Aguirre D, GordilloMoscoso A, Chalita-Williams C: Use of statins for the treatment of spontaneous intracerebral hemorrhage: results of a pilot study. Cent Eur Neurosurg. 2009, 70:15-20. 10.1055/s-0028-1082064

10. Endres M, Nolte CH, Scheitz JF: Statin treatment in patients with intracerebral hemorrhage. Stroke. 2018, 49:240-246. 10.1161/STROKEAHA.117.019322

11. Naval NS, Abdelhak TA, Zeballos P, Urrunaga N, Mirski MA, Carhuapoma JR: Prior statin use reduces mortality in intracerebral hemorrhage. Neurocrit Care. 2008, 8:6-12. 10.1007/s12028-007-0080-2

12. FitzMaurice E, Wendell L, Snider R, et al.: Effect of statins on intracerebral hemorrhage outcome and recurrence. Stroke. 2008, 39:2151-2154. 10.1161/STROKEAHA.107.508861

13. Eichel R, Khoury ST, Ben-Hur T, Keidar M, Paniri R, Leker RR: Prior use of statins and outcome in patients with intracerebral haemorrhage. Eur J Neurol. 2010, 17:78-83. 10.1111/i.1468-1331.2009.02747.x 
14. Leker RR, Khoury ST, Rafaeli G, Shwartz R, Eichel R, Tanne D: Prior use of statins improves outcome in patients with intracerebral hemorrhage: prospective data from the National Acute Stroke Israeli Surveys (NASIS). Stroke. 2009, 40:2581-2584. 10.1161/STROKEAHA.108.546259

15. Gomis M, Oisa A, Rodriguez-Campello A, et al.: Outcome of intracerebral haemorrhage patients pre-treated with statins. Eur J Neurol. 2010, 17:443-448. 10.1111/j.1468-1331.2009.02838.x

16. Romero FR, Bertolini EF, Veloso VN, Venturini L, Figueiredo EG: Outcomes from intracerebral hemorrhage among patients pre-treated with statins. Arq Neuropsiquiatr. 2011, 69:452-454. 10.1590/s0004282x2011000400008

17. Dowlatshahi D, Demchuk AM, Fang J, Kapral MK, Sharma M, Smith EE: Association of statins and statin discontinuation with poor outcome and survival after intracerebral hemorrhage. Stroke. 2012, 43:15181523. 10.1161/STROKEAHA.111.645978

18. King NK, Tay VK, Allen JC, Ang BT: Prior statin use has no effect on survival after intracerebral hemorrhage in a multiethnic Asian patient cohort. Acta Neurochir Suppl. 2012, 114:343-346. 10.1007/978-3-7091-09564_66

19. Mustanoja S, Strbian D, Putaala J, et al.: Association of prestroke statin use and lipid levels with outcome of intracerebral hemorrhage. Stroke. 2013, 44:2330-2332. 10.1161/STROKEAHA.113.001829

20. Flint AC, Conell C, Rao VA, et al.: Effect of statin use during hospitalization for intracerebral hemorrhage on mortality and discharge disposition. JAMA Neurol. 2014, 71:1364-1371. 10.1001/jamaneurol.2014.2124

21. Winkler J, Shoup JP, Czap A, Staff I, Fortunato G, McCullough LD, Sansing LH: Long-term improvement in outcome after intracerebral hemorrhage in patients treated with statins. J Stroke Cerebrovasc Dis. 2013, 22:541-545. 10.1016/j.jstrokecerebrovasdis.2013.06.015

22. Siddiqui FM, Langefeld CD, Moomaw CJ, et al.: Use of statins and outcomes in intracerebral hemorrhage patients. Stroke. 2017, 48:2098-2104. 10.1161/STROKEAHA.117.017358 